the non-calculous cholecystitis is the so-called "strawberry gall-bladder," in which the mucous membrane has the appearance of a ripe strawberry.

Cholecystitis without stones is usually accompanied by habitual tenderness in the region of the gall-bladder. As a rule, colic is not a prominent symptom, and if present is not so severe in character as that accompanying gall-stones. There is often a mild constitutional disturbance, and in some instances, for months at a time, there will be a slight elevation of evening temperature (99 to $100 \mathrm{~F}$.), but without marked loss of flesh; the patient complaining of lassitude and other symptoms of a chronic mild toxemia.

Non-calculous cholecystitis occurs in several forms; the two most frequent are the following: 1. The distended thin-walled gall-bladder contains several ounces of colon-infected bile, often with a foul odor. 2. The -wall of the gall-bladder is thickened and white, often adherent and contains a thick, tarry material. In both of these forms the lymph-glands along the common ducts are enlarged. In one of our cases of cholecystitis of long standing (twenty years or more) these enlarged glands had become calcareous, producing sufficient pressure on the common duct to cause chronic jaundice.

Complications were found in more than two-thirds of the patients on whom we operated for gall-stones. Stones were found in the common duct in 492 cases, while serious complications involving the liver, duodenum, transverse colon, etc., were the rule. Carcinoma was found associated with gall-stones in 85 cases (2.25 per cent.). We have removed a number of gall-bladders for carcinoma, and in several instances have excised a piece of the adjacent liver. In cases in which the carcinomatous condition of the gall-bladder was sufficiently advanced to diagnose at the time of operation, the patients did not survive one year, but in a number of cases an early carcinoma was removed accidentally, so to speak, in removing thick-walled functionless wallbladders, and five of these patients are alive and well at periods of from two to six years after operation.

Gall-stones are foreign bodies. Why delay operating until complications occur? In our experience simple operation for uncomplicated gall-stones has had a nnortality of less than 0.5 per cent., and this might be called accidental, since it was due to the condition of the patient, rather than to the operation.

Early operation is relatively safe and the gall-bladder may be saved in a condition for future function. Fatalities can be traced in almost every instance to delay. That temporary palliation can be procured with nonoperative measures cannot be denied (Carlsbad, etc.), but the cure of the patient can only be brought about by surgical measures.

In this connection may be noted the wide difference of opinion between the British and American observers, on the one hand, who urge early operation, and those continental observers on the other hand, who advocate delay in operation. One of the most prominent of the continental surgeons draws conclusions which may be epitomized as follows: 1 . Stones in the gall-bladder do not require operation until they have produced marked (hanges in the wall of the gall-bladder, i. e., formation of tumor, etc. 2. A long incision shonld alwars be made in operating for gall-stone disease. 3. The jleal operation is removal of the gall-bladider. 4. Drainage of the hepatic ducts will usually be necessary. In reply the advocates of early operation would sav: 1 . In operating before gross changes are manifest the danger is minimized and the mortality is small. 2. In operating early an incision longer than is necessary to introduce the hand for exploration is not often required, and prolonged convalescence and the danger of hernia is avoided. 3 . In early operations it is not necessary to remove the gall-bladder, and this valuable organ is saved. 4. Early operation relieves the patient before deep-seated infections occur which necessitate drainage of the liver ducts.

\section{HOOKWORM DISEASE}

PREVALENCE, HARMFUL RESULTS AND TREATMENT *

\section{F. STROSNIDER, M.D.}

Golusboro, N. c.

\section{PREVALENCE OF HOOKWORM DISEASE}

Hookworm disease prevails in tropical and semitropical regions. Its distribution is now being studied in many parts of the world, and especially in our South; in every southern state a number of physicians are spending their entire time collecting data on its prevalence, and endeavoring as best they may to effect its eradication. Already it is known that the disease exists in Virginia on the Potomac River, in Florida, in Texas and in the intervening states, including North Carolina, South Carolina, Georgia, Alabama, Mississippi, Louisiana, Arkansas and Tennessee.

The following results of investigations which have been made will give some idea of the degree of infection among our people. Chamberlin in 1909 examined a number of southern recruits and found 60 per cent. of them infected. Later Siler in examining 105 recruits found ninety-three infected, which is 88.5 per cent. In North Carolina during the past summer the following examinations have been made with results as indicated (Figures collected by Dr. J. A. Ferrell, director of the North Carolina campaign) :

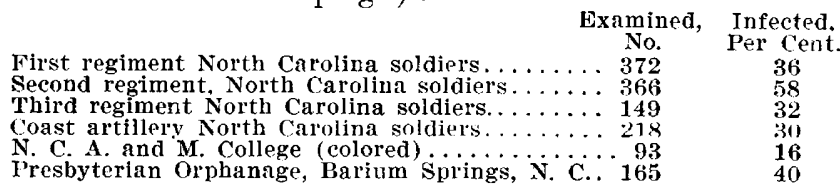

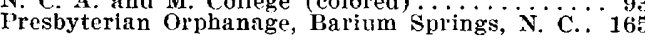

Odd Fellows' Orphanage, Goldsboro, N. C. :

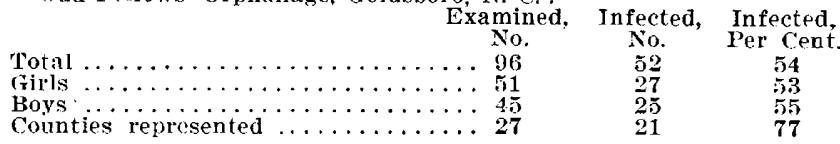

Ifethodist Orphanage, Raleigh, N. C.

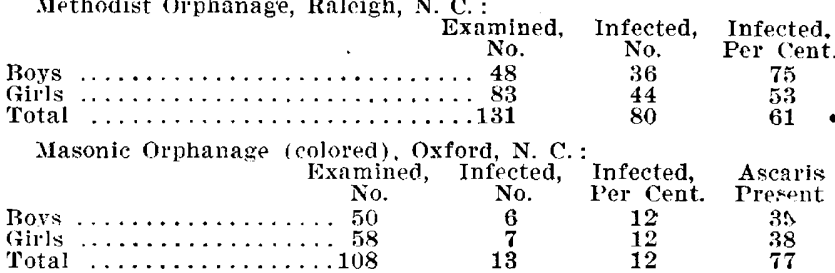

'The children here, as in the other orphanages, range in age from 6 to 18 years.

Dr. B. W. Page, assistant to the Secretary for Horkworm Disease to North Carolina Board of Health, had 394 children at the Baptist Orphanage examined and found 38 per cent. of them infected. Dr. C. L. Pridgen, is also an assistant in the North Carolina Hookworm Campaign, working in the western section of the state. His examinations of school children showed the following: Fifty-four specimens from Mitchell and Yancey

* Read at the medical society meeting of the Fourth District of North Carolina. 
countics showed twenty-eight infections with hookworm, and twenty-five with Ascaris lumbricoides. Twenty-eight examinations in McDowell County showed twenty-eight infections, or 73 per cent., and in the town of Marion in the same county, 54 per cent. of infection was found in thirty-five examinations.

Serving the North Carolina Hookworm Commission in same capacity as the two physicians last named and working in eastern North Carolina, I found the infection running somewhat higher than in the central and western sections of the state. In the Kinston High School fifty-one were examined; twenty-eight or 55 per cent. of them showed hookworm infection. At Magnolia in I uplin County, seven school children were examined and six were found infected. At Faison, in the same county, twenty-four people were examined whose ages ranged from nine to forty years, and thirteen were found infected. Other schools showed the following:

Wayne County, Eureka, 6 examined, 4 infected, or $662 / 3$ per cent. Wayne county, 21 examined, 19 infected. or 90.5 per cent. Wayne County,

19 examined, 13 infected, or 68.4 per cent.

Moreover, Dr. K. A. Price, of Hickory, had fifty-six male college students examined and found nine infected. Examinations of school children are now being made in large numbers and the results of these will soon be obtainable. At this time only the figures above given are in my possession. The figures given tend to show that the disease exists in all sections of the state, but that it is more prevalent in the eastern counties. This may be due in part to the fact that the soil is largely sandy and level, factors favoring the propagation of the hookworms; in part to the fact that the negroes, who are regarded as extensive worm-carriers, though they suffer but little from the disease, are more densely settled. in the east than in the west, and in part perhaps to the fact that the counties in the tide-water belt have longer and warmer summers than the mountain counties.

Further information on the prevalence of the disease in the state mav. it would seem, soon be obtained from our general practitioners. During the first three months of 1910 they sent to the state laboratory only seventy specimens for examination. During the second quarter, which ended June 30 , they sent in 486 specimens, and during the three months ending September 30, 1910, they sent in 2,421 specimens. If their interest in the work continues to grow with such rapidity the distribution of the discase may be known to a certainty.

\section{HARMFUL RESULTS}

The thorough discussion of the far-reaching harm resulting from hookworm infection would be too lengthy for the present paper. Dr. H. F. Harris, secretary to the Georgia State Board of Health. says: "In no other disease does the rictim suffer so long; in no other condition is he for such a period a menace to those about him; in no other malady of such gravity is treatment so rapidly and surely successful." The more immediate results of the disease are of vital importance.

Perhaps the harm produced by the worms will be more easily explained if first a word is given to the characteristics of the worms. They are blood-sucking parasites, varying in length from one-fourth to one-half inch; in numbers they range from less than a hundred to more than four thousand in a single victim of the disease. They inhabit the small intestine and attach themselves by their mouths, which are supplied with powerful muscles, teeth and sucking surfaces, to the mucous membrane. They move from place to place in fecding on the blood they suck from the flesh, each time making a new wound. The loss of blood resulting flom their action is of greatest importance. Much blood is drawn directly into the bodies of the worms, but a more important loss perhaps occurs through the minute hemorrhages from the wounds which they make. So great is this latter loss in some instances that the blood in the stool of the victim may be recognized by the naked eye; if it is less, it may be detected by the usual chemical tests. Ordinarily the loss of blood would be somewhat dependent on the number of invading parasites. There are cases, however, in which the number of worms present seems to account only in a remote degree for the symptoms produced. 'The patient's age, his constitution, his digestive system, and his power to manufacture new blood are points which determine, to some extent, the amount and effect of the loss of blood.

A second harmful result is the poisoned system. The worms are supposed to secrete and inject into the flesh a hemolytic poison, which, moreover, prevents the coagulation of blood in the wounds, from which, therefore, much blood is lost by oozing. Moreover, the excrementitious products from the worms are poisonous, and since the wounds offer points for easy entrance of pathogenic organisms, in many instances the wounds are followed by suppuration, and poisons from such places, when absorbed, increase the general poisoned condition of the patient's svstem.

In addition to the loss of blood and the poisoning of the srstem should be mentioned the damage done to the intestine. The numerous bites, together with the poison, give rise to a chronic catarrhal inflammation of the intestine. This impairs digestion and causes tenderness in the epigastric region. To this may be added what is really of sufficient impertance for separate consideration. With much denuded and diseased mucosa exposed, disease organisms, particularly tubercle bacilli, and typhoid bacilli, which find entrance into the intestinal canal with food or water, easily pass into the lrmphatic or blootcurrents and ultimately overwhelm the defenseless victim with the disease they produce.

The secondary changes which result from the hookworms will be best understood by discussing them in connection with the symptoms of the diseasc. Anemia, with its important effects on all nutritive and metabolic processes, accounts for most of the striking features presented by a hookworm victim, such especially as physical and mental weakness. It also predisposes the person to other diseases and in many instances the ensuing complication is regarded as the primary cause of the death of the person.

For convenience of description I shall classify the disease, according to its harmful results, into four divisions: namely, mild, medium, severe, and very severe.

Mild Cases. - In the mild cases we have, at present, no recognizable symptoms, and ther are only diagnosed by the microscope. Though I have found in adults mill digestive disturbances, such as slight reduction of digestive ability, tenderness, pain, and discomfort in the epigastrium, accompanied by nervousness. (This is by no means constant.) Sometimes only a small number of worms are present; again 200 to 300 worms may bo present and not produce recognizable symptoms (Dock and Bass).

iledium.-Cases of medium severity have little pallor or have a slightly dirty, yellow tinge of the skin in whites, and a slight pastiness in mulattoes. There is a reruction in the normal amount of perspiration; the appetite is frequently excessive. There may be uneasi- 
ness, pain or tenderness in the epigastrium, frequent attacks of meteorism, slight dyspnea on exertion, palpitation of the heart, dizziness, headache, lack of attention to details, and a little stupidity. The muscles are flabby, and weakness and indisposition to labor exists (laziness). The hemoglobin of such persons generally is between 60 ner cent. and normal. Joint pains, especially in the sternum, constitute a frequent symptom. The temperature may range from 99 to $101 \mathrm{~F}$.

Severe Cases. - Here all symptoms noted under medium cases are more prominent. The pallor is definite, the mucous membranes are paler than normal, and where the same form a juncture with the skin it often fades away so gradually as to be imperceptible, the skin is quite dry, and sweating is rare. The appetite. may be exaggerated or diminished. Nausea is frequent. The tongue is coated and often flabby and enlarged. Tenderness of the epigastrium, with spontaneous pain on gentle pressure at times, is present. (In sixty inspections followed by microscopic examination, this sign has proved positive in every one, being the most constant, most suggestive and most marked of all signs or symptoms.) Breathlessness is easily brought about by the slightest exertion. There is often a hemic murmur of the heart. There may be much pain in the sternum and chest, with a feeling of weakness of the knees and legs. There is much dizziness, especially on sudden changes of position, noise in the ears and frequent headaches. The mental condition is decidedly depressed, and the patient is quite passive to his surroundings, and presents a stupid appearance. The patellar reflex may be either exaggerated or diminished. Patients complain of cold and tingling of the feet, which frequently go to sleep; the muscles are flabby and may be slightly painful; joint pains are so common that a diagnosis of rheumatism is often made. Work is performed with great effort and is deficient in both quality and quantity. In about ten days after ground-itch appears the patient usually develops a sore throat, a slight bronchitis, and a temperature ranging from $99 \mathrm{~F}$. to $102 \mathrm{~F}$. The skin is waxy, tallow or tan color. Edema of the face may be present. The hemoglobin is apt to be between 45 and $7_{0}$ per cent.

Very Severe Cases.-A case in this stage of the disease may terminate fatally at any time. The skin is tan or tallow-colored. Fdema of the feet and ankles alone, or sometimes extreme anasarca may be present. The anpetite may be slight or enormous. Nausea and vomiting are frequent. Ascites mav be alarming. Diarrhea often alternates with constipation. There is distressing dyspnea on slightest exertion, severe palpitation of the heart, pericordial pain, rapid, weak and compressible pulse, and often dilatation of the heart; marked pulsation of the ressels of the neck; dizziness and tinnitus aurium are extreme; patellar reflex is abolished. In men impotence is an almost invariable symptom, and amenorrhea in women. The intellect is dull and the patient scems confused and stupid. The facial expression. is anxious and stupid. There is a tendency to dilatation of the pupil, and blurred vision exists. The urine has a low specific gravity, is abunclant in quantity, and usually contains no albumin. The muscles are flabby, sore and painful. Extreme weakness is observed and the patient $j s$ incapacitated for labor. There is often irregular fever. with intervals of subnormal temperature.

Acute hookworm disease may occur without previous infection, or an acute attack may supervene on a chronic infection. These acute attacks occur in the spring and summe", and their symptoms vary with the severity of the infection. Should sufficient infection occur, there will be a sore throat, and bronchitis, nausea or vomiting, a chill and diarrhea, epigastric pains or colic, rapid anemia, and elevation of temperature as high as 101 or $102 \mathrm{~F}$. One difference between acute and chronic cases is that the skin is waxy white with severe anemia, whereas in chronic cases, with the same amount of anemia there is a more yellow tinge ("tallow-faced"). Children with acute hookworm disease have not the "potbelly" present in so many of the chronic, severe cases. In very severe infections, as in Claud A. Smith's experimental case, great swelling occurs on the second and third days. Loose has noticed red lines running up the leg-probably a lymphangitis.

Nervous System.-Insomnia and night terrors are quite common, because of motion of the worms. Somnolence often occurs, and the patient keeps awake with difficulty. Lack of ambition and lassitude are noticeable. After administration of thymol the temperature frequently rises to 102 or $104 \mathrm{~F}$., usually subsiding in twelve hours. I offer the following explanation of this phenomenon: When the thymol comes in contact with the worms they become very active; the thymol causes them to vomit and purge most of their ingested blood (Bass); at the same time they eliminate a large amount of toxin; much blood escapes from their points of attachment. Therefore, if the hookworm toxin acts as a direct poison, and the blood absorption acts as an auto-intoxicant, I feel that a rise in temperature would be accounted for.

\section{QUESTIONS BFARING ON POSSIBLE ADDITIONAL HARMFUL, RESULTS}

1. May not many cases of neurasthenia and hysteria be attributable to hookworm disease?

2. Could not such perversions, among hookworm patients, as disobedience, profanity, lying, stealing, drinking, forging, and sexual perversions be attributable to hookworm disease, provided recovery followed cure of the hookworms?

3. Is not chlorosis or "green-sickness," in many cases a result of uncinariasis?

4. Is it not probable that a number of cases of defective eyes and ears, and diseased tonsils and adenoids could be attributed to the same cause?

5. Is it not true that many abortions and miscarriages can be attributed to anemia resulting from hookworm disease?

6. Does not observation show that, as Dr. Nicholson of Onslow County says, whereas the average eclampsia with first child in the United States is 1 to 250 , in women suffering with hookworm disease the average is 1 to 50 .

7. Is it not probable that many women die from shock during labor as a result of hookworm disease?

8. Is it not a fact that, as a result of this disease, many women cannot furnish sufficient milk to nourish their babies?

9. Is it not a fact that hookworm disease is the cause of more amenorrhea, dysmenorrhea, and scanty and irregular menstruations, than any other disease in our South?

10. Is it not a fact that a hookworm patient, pregnant, quite often suffers with a severe dropsy, and often after delivery may run an irregular temperature for some time and fail to react to tonics or quinin? 


\section{TREATMENT}

Externat.-Ground-itch in the papular or vesicular stage may be treated with a 5 per cent. salicylic acid suspended in collodion. This usually cuts the attack of ground-itch to one or two days. During the pustular state, the wound should be cleansed and cauterized with silver nitrate and then a dry dressing of 5 per cent. boric acid and zine oxid ointment applied twice daily.

When great swellings occur hot applications should be used. To allay itching and prevent secondary infection a combination 5 per cent. zinc oxid and salicylic acid ointment applied locally, twice daily, is recommended. It is important to keep the foot bandaged or covered to prevent scratching or rubbing.

Internal.-Treatment should be begun on the fifteenth day from date of the ground-itch and repeated once. The same treatment should be instituted after an attack of ground-itch as if eggs were present in the stools, thus getting rid of the worms before they reach maturity and have done much harm. The stools should be examined at the end of six and twelve weeks. from the attack of ground-itch for eggs of any worm that may have escaped previous treatment.

Preliminary.-The first step is to remove the protecting chyme and mucus by a large dose. of saltsRochelle, Epsom or Glauber's-four hours after the last meal. Sodium sulphate dissolves and removes the intestinal mucus best. 'The drugs used to kill or expel the worms are thymol, betanaphthol and male-fern.

Dose of Thymol.- The dose for children should be in proportion to the sizc. apparent age, and physical condition: Ashford and King give the following table of doses, to be regulated, in the case of children, rather by size than by actual age.

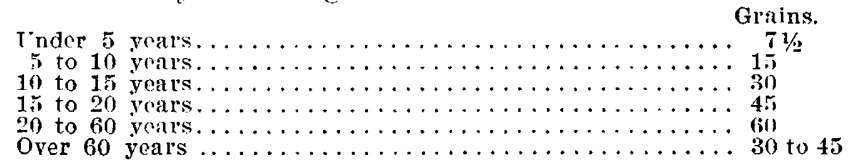

I have heen giving about 2 grains per year up to the age of 30 , the same to be administered in two equal doses two hours apart. Unfavorable conditions, such as great weakness, extreme anemia, diarrhea, cardiac depression, pregnancy, and dropsy, require a smaller dose.

Mode of Administration of Thymol.-This should be given in two or three broken doses to prolong its presence in the upper bowel. Because of its tendency to pack together under pressure or in the mucus in the intestine, equal parts of sugar of milk or some other soluble sul)stance should be mixed with it. To prevent its burning of the mouth and throat, it should be given in cachets, wafers, or capsules; it may also be given suspended in rither mucilage or syrup of acacia or some simple srrup. The patient should lic on the right side and refrain from drinking much water, to prevent vomiting. After two or three hours another hrisk purgative of salts (never castor oil) should be given to prevent its ahsorption. The patient should remain in hed until the thymol is moved off. Either the bed-pan or the commode should be used in the room to avoid possible fainting.

Diet During Thymol Treatment.-'To keep the alimentary canal clean, no food or drink, except water, should be allowed from the time the preparatory purgative is given until the purgative following the thymol las acted well. If the patient is weak or faint, or if the burning in the stomach is excessive, a little warm coffee may be allowed. The diet need not be restricted after several free movements have occurred following the postthymol purgative, unless there is some special indication. The treatment should be repeated once a week until examination of the stool, washed through a piece of cheese-cloth, shows no worms, or the microscopic exam. ination shows no eggs.

Saturday evening and Sunday is the most convenient time to administer the treatment to working people and school children. The salts purge should be given Saturday evening and thymol begun early Sunday morning. For example, Saturday at 8-9 p. m. sodium sulphate may be given; Sunday at 6 a. m., thymol; Sunday at 8 a. m. thymol again; at 10 a. m., sodium sulphate. Until $4 \mathrm{p}$. m. the patient should not eat, or drink anything except water, and but little of that.

Caution.-Oils, fats (milk or butter), patent medicines, or beverages containing alcohol should not be allowed during thymol treatment. Thymol may affect the patient in one of three different ways: by intoxication, by irritation of the kidney, and by irritation of the stomach and intestine. The effects of absorbing a large amount of thymol are depression, headache, weakness, dizziness, tinnitus, nausea, unconsciousness, rapid, weak pulse, and sometimes profuse sweating and subnormal temperature (about $1 / 2 \mathrm{gr}$. of thymol is soluble to the ounce of water). The effect of thymol on the gastrointestinal canal is occasional epigastric pain, nausea and vomiting, and more or less diarrhea. Therefore gastritis, and diarrhea or dysentery are contra-indications. About half a dose should be given to a very weak subject the first time.

Treatment of Toxic Symptoms.-Cardiac stimulants should be given, morphin and atrepin, strychnin, digitalin or ergot, subcutaneously, and hot coffe per rectum. Alcoholic stimulation should be avoided. The patient should be kept recumbent. Hot blankets should be applied to the body and a warm water enema used.

301 South Williams Street.

\section{SANITARY CONSIDERATION OF HEALTH RESORTS}

HAROLD B. WOOD, M.D., C.S.

JACKsos, MIss.

The recommendation of health resorts is frequently expected of physicians who unconsciously assume the risk, little thinking what effects, both good and bad, the individual town or hotel may have on its guests. Convalescents, travellers and vacationists visit the health and summer resorts unsuspecting of any danger or with the confidence that the conditions are as the physician assumes or expects. The mere mention or naming of a resort by a physician is considered a recommendation and a therapentic direction to be followed as any other therapeutic order. Opinions of resorts must be formed from personal observation, from description by others, or advertisement. There is an avoidance of the whole truth in many advertisements, yet these misstatements or evasions are accepted without question, and serving as the directing force, may ultimately end disastrously to the guests and finally to the resorts.

$\mathrm{Had}$ one physician, referring to a western town as a well-known health resort, known its true condition and the possibility of contracting typhoid fever there, he would have pronounced it anything but a health resort. 